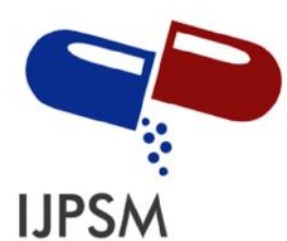

Dr. M.Kannadasan et al, Int. Journal of Pharmaceutical Sciences and Medicine (IJPSM), Vol.5 Issue. 12, December- 2020, pg. 46-58

ISSN: 2519-9889

Impact Factor: 3.426

\title{
A REVIEW: NANO PARTICLE DRUG DELIVERY SYSTEM
}

\author{
Dr. M.Kannadasan ${ }^{1}$; Prem Kumar Bichala ${ }^{2}$; Abhishek Agrawal'; Swapna Singh ${ }^{2}$ \\ ${ }^{1}$ School of Pharmaceutical Sciences, Pratap University, Jaipur, Rajasthan, m.kannadasan@ pratapuniversity.in \\ ${ }^{2}$ School of Pharmaceutical Sciences, Pratap University, Jaipur, Rajasthan, prem.kumar@ pratapuniversity.in \\ ${ }^{2}$ School of Pharmaceutical Sciences, Pratap University, Jaipur, Rajasthan, abhishek.agrwal@ pratapuniversity.in \\ ${ }^{2}$ School of Pharmaceutical Sciences, Pratap University, Jaipur, Rajasthan, swapnasingh112@ gmail.com
}

DOI: 10.47760/ijpsm.2020.v05i12.008

\begin{abstract}
In the recent past, the targeted drug delivery has gained more attention for various advantages. Amongst the plethora of Avenues explored for targeted drug delivery. Nanoparticles are particulate dispersions or solid particles with a size in the range of 10-1000nm. The drug is dissolved, entrapped, encapsulated or attached to a nanoparticle matrix. Depending upon the method of preparation, nanoparticles, nanospheres or nanocapsules can be obtained. The major goals in designing nanoparticles as a delivery system are to control particle size, surface properties and release of pharmacologically active agents in order to achieve the sitespecific action of the drug at the therapeutically optimal rate and dose regimen. Present review reveals the methods of preparation, characterization and application of several nanoparticulate drug delivery systems.

KEYWORDS: Nano particle drug delivery system, nanospheres, nanocapsules.
\end{abstract}

\section{INTRODUCTION ${ }^{1,2,4}$}

Nanoparticles are defined as particulate dispersions or solid particles with a size in the range of $10-1000 \mathrm{~nm}$. The drug dissolved, entrapped, encapsulated or attached to a nanoparticles matrix. Depending upon to the method of preparation, nanoparticles, nanospheres or nanocapsules can be obtained. Nanocapsules are systems in which the drug is confined to a cavity surrounded by a unique polymer membrane, while nanospheres are matrix systems in which the drug is physically and uniformly dispersed. In recent years, biodegradable polymeric nanoparticles, particularly those coated with hydrophilic polymer such as poly (ethylene glycol) (PEG) known as long-circulating particles, have been used as potential drug delivery devices because of their ability to circulate for a prolonged period time target a particular organ, as carrier of DNA in gene therapy, and their ability to deliver proteins, peptides and genes.

Many biomaterials, primarily polymer- or lipid-based, can be used to this end, offering extensive chemical diversity and the potential for further modification using nanoparticles. The particularly large surface area on the nanoparticles presents diverse opportunities to place functional groups on the surface. Particles can be created by expanding or contracting with changes in temperature or $\mathrm{pH}$, or interact with anti-bodies in special ways to provide rapid ex-vivo medical diagnostic tests.

More practical design extensions have been made in combining inorganic materials with polymers and in combining different classes of polymers together in nanoparticle form. A whole host of new types of polymer particles could be designed into reality with the recent advances in chemistry, processing techniques, and analytical instrumentation. For example, now we have particles that are hollow, multi-lobed, conductive, thermo responsive, magnetic, 


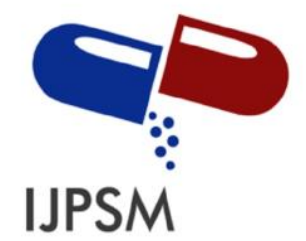

\section{Dr. M.Kannadasan et al, Int. Journal of Pharmaceutical Sciences and Medicine (IJPSM), Vol.5 Issue. 12, December- 2020, pg. 46-58}

ISSN: 2519-9889

Impact Factor: 3.426

functionalized with reactive groups on the surface, and $\mathrm{pH}$ responsive. This could be applied into floating carrier, multiparticulate drug delivery, dual core or multiple layering drugs and more.

Polymeric nanoparticles have been produced for decades for use in a variety of high performance materials such as high impact resistant polymers and specialty coatings for these purposes. Advanced analytical techniques and computer simulations of the events occurring during particle formation allow us to measure structure and develop control strategies to produce structured particles. Our ability to develop new control process strategies such as modified the of carrier shape, chemical composition, internal structure, and morphology of the nanoparticles so as to develop new levels of product performance in the targeted drug delivery system.

Nanotechnology, the term derived fromGreek word 'Nano', meaning dwarf, applies the principles of engineering, electronics, physical and material science \& manufacturing at a molecular and supra-micron level. Nanoparticles are defined as particulate dispersions or solid particles with a size in the range of $10-1000 \mathrm{~nm}$. The drug is dissolved, entrapped, encapsulated or attached to a nanoparticle matrix. Depending upon the method of preparation, nanoparticles, nanospheres or nanocapsules can be obtained. Nanocapsules are systems in which the drug is confined to a cavity surrounded by a unique polymer membrane, while nanospheres are matrix systems in which the drug is physically and uniformly dispersed.

Types of Nanoparticles [2,3]

The classes of nanoparticles listed below are all very general and multi-functional; however, some of their basic properties and current known uses in nanomedicine are described here.
1) Solid lipid nanoparticles (SLNs)
2) Liposomes
3) Nanostructured lipid carriers (NLC)
4) Fullerenes
5) Nanoshells
6) Quantum dots (QD)
7) Super paramagnetic nanoparticles

\section{Solid lipid nanoparticles (SLNs)}

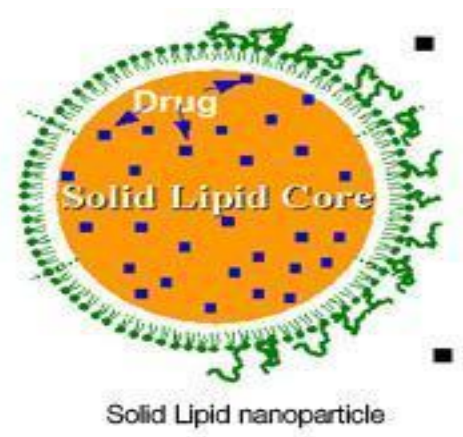

Figure 1: Solid lipid nanoparticles

SLNs mainly comprise lipids that are in solid phase at the room temperature and surfactants for emulsification, the mean diameters of which range from $50 \mathrm{~nm}$ to $1000 \mathrm{~nm}$ for colloid drug delivery applications SLNs offer unique properties such as small size, large surface area, high drug loading, the interaction of phases at the interfaces, and 


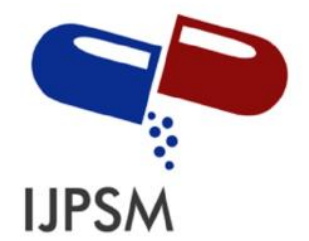

\section{Dr. M.Kannadasan et al, Int. Journal of Pharmaceutical Sciences and Medicine (IJPSM), Vol.5 Issue. 12, December- 2020, pg. 46-58}

ISSN: 2519-9889

Impact Factor: $\mathbf{3 . 4 2 6}$

are attractive for their potential to improve. Typical methods of preparing SLNs include spray drying high shear mixing ultra-sonication and high pressure homogenization (HPH) Solid lipids utilized in SLN formulations include fatty acids (e.g. palmitic acid, decanoic acid, and behenic acid), triglycerides (e.g. trilaurin, trimyristin, and tripalmitin), steroids (e.g. cholesterol), partial glycerides (e.g. glyceryl monostearate and gylceryl behenate) and waxes (e.g. cetyl palmitate). Several types of surfactants are commonly used as emulsifiers to stabilize lipid dispersion, including soybean lecithin,phosphatidylcholine, poloxamer 188, sodium cholate, and sodium glycocholate Advantages of these solid lipid nanoparticles (SLN) are the use of physiological lipids, the avoidance of organic solvents in the preparation process, and a wide potential application spectrum (dermal, oral, intravenous).

\section{Liposomes}

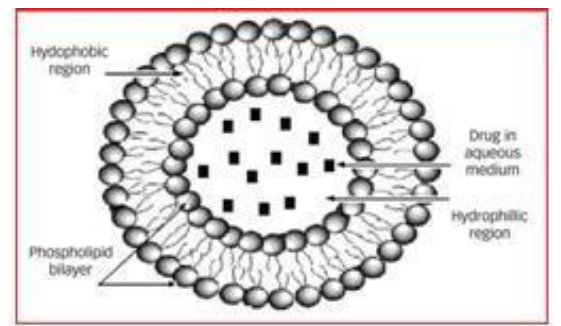

Figure 2: Liposomes

Liposomes are vesicular structures with an aqueous core surrounded by a hydrophobic lipid bilayer, created by the extrusion of phospholipids. Phospholipids are GRAS (generally recognized as safe) ingredients, therefore minimizing the potential for adverse effects. Solutes, such as drugs, in the core cannot pass through the hydrophobic bilayer however hydrophobic molecules can be absorbed into the bilayer, enabling the liposome to carry both hydrophilic and hydrophobic molecules.

The lipid bilayer of liposomes can fuse with other bilayers such as the cell membrane, which promotes release of its contents, making them useful for drug delivery and cosmetic delivery applications. Liposomes that have vesicles in the range of nanometers are also called nanoliposomes. Liposomes can

vary in size, from $15 \mathrm{~nm}$ up to several $\mathrm{lm}$ and can have either a single layer (unilamellar) or multiple phospholipid bilayer membranes (multilamellar) structure. Unilamellar vesicles (ULVs) can be further classified into small unilamellar vesicles (SUVs) and large unilamellar vesicles (LUVs) depending on their size range.

\section{Nanostructured lipid carriers (NLC)}

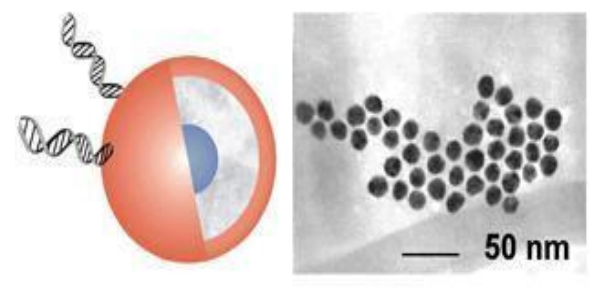

Figure 3: Nanostructured lipid carriers (NLC) 


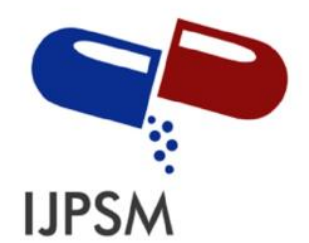

\section{Dr. M.Kannadasan et al, Int. Journal of Pharmaceutical Sciences and Medicine (IJPSM), Vol.5 Issue. 12, December- 2020, pg. 46-58}

ISSN: 2519-9889

Impact Factor: $\mathbf{3 . 4 2 6}$

Nanostructured Lipid Carriers are produced from blend of solid and liquid lipids, but particles are in solid state at body temperature. Lipids are versatile molecules that may form differently structured solid matrices, such as the nanostructured lipid carriers (NLC) and the lipid drug conjugate nanoparticles (LDC) that have been created to improve drug loading capacity. The NLC production is based on solidified emulsion (dispersed phase) technologies.

NLC can present an insufficient loading capacity due to drug expulsion after polymorphic transition during storage, particularly if the lipid matrix consists of similar molecules. Drug release from lipid particles occurs by diffusion and simultaneously by lipid particle degradation in the body. In some cases it might be desirable to have a controlled fast release going beyond diffusion and degradation. Ideally this release should betriggered by an impulse when the particles are administered. NLCs accommodate the drug because of their highly unordered lipid structures. A desired burst drug release can be initiated by applying the trigger impulse to the matrix to convert in a more ordered structure. NLCs of certain structures can be triggered this way. NLCs can generally be applied where solid nanoparticles possess advantages for the delivery of drugs.

\section{Fullerenes}

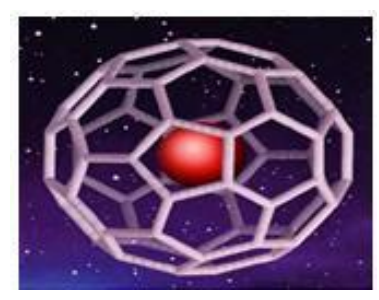

Figure 4: Fullerenes

A fullerene is any molecule composed entirely of carbon, in the form of a hollow sphere, ellipsoid, or tube. Spherical fullerenes are also called buck balls, and cylindrical ones are called carbon nanotubes or buck tubes. Fullerenes are similar in structure to the graphite, which is composed of stacked grapheme sheets of linked hexagonal rings, additionally they may also contain pentagonal (or sometimes heptagonal) rings to give potentially porous molecules. Buckyballclusters or buck balls composed of less than 300 carbon atoms are commonly known as endohedral fullerenes and include the most common fullerene, buckminsterfullerene, C60.

\section{Nano shells}

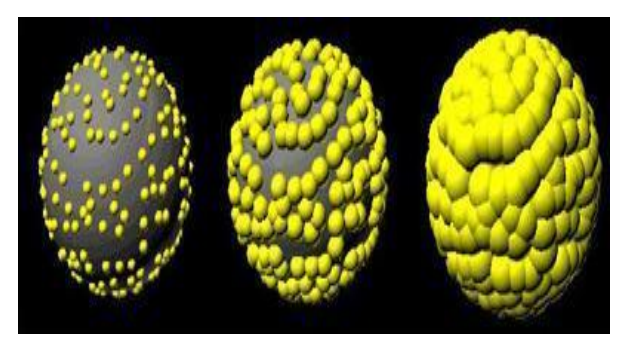

Figure 5: Nano Shells 


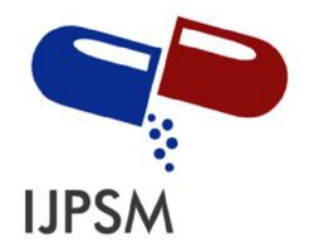

\section{Dr. M.Kannadasan et al, Int. Journal of Pharmaceutical Sciences and Medicine (IJPSM), Vol.5 Issue. 12, December- 2020, pg. 46-58}

ISSN: 2519-9889

Impact Factor: $\mathbf{3 . 4 2 6}$

Nanoshells are also notorious as core-shells, nanoshells are spherical cores of a particular compound (concentric particles) surrounded by a shell or outer coating of thin layer of another material, which is a few 1-20 nm nanometers thick Nanoshell particles are highly functional materials show modified and improved properties than their single component counterparts or nanoparticles of the same size. Their properties can be modified by changing either the constituting materials or core-to-shell ratio Nanoshell materials can be synthesized from semiconductors (dielectric materials such as silica and polystyrene), metals and insulators. Usually dielectric materials such as silica and polystyrene are commonly used as core because they are highly stable. Metal nanoshells are a novel type of composite spherical nanoparticles consisting of a dielectric core covered by a thin metallic shell which is typically gold. Nanoshells possess highly favorable optical and chemical properties for biomedical imaging and therapeutic applications. Nanoshells offer other advantages over conventional organic dyes including improved optical properties and reduced susceptibility to chemical/thermal denaturation. Furthermore, the same conjugation protocols used to bind biomolecules to gold colloid are easily modified for nanoshells. When a nanoshell and polymer matrix is illuminated with resonant wavelength, nanoshells absorb heatand transfer to the local environment. This causes collapse of the network and release of the drug. In core shell particles-based drug delivery systems either the drug can be encapsulated or adsorbed onto the shell surface. The shell interacts with the drug via a specific functional group or by electrostatic stabilization method. When it comes in contact with the biological system, it directs the drug. In imaging applications, nanoshells can be tagged with specific antibodies for diseased tissues or tumors.

\section{Quantum dots (QD)}

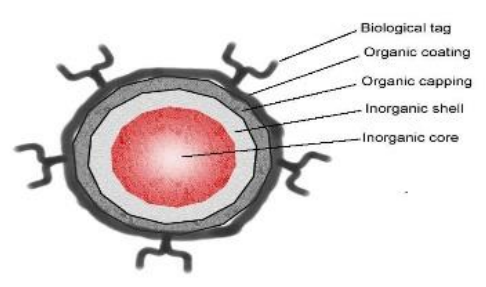

Figure 6: ${ }^{20}$ Q $\mathrm{Qm}$ uantum dots (QD)

The quantum dots are semiconductor nanocrystals and core shell nanocrystals containing interface between different semiconductor materials. The size of quantum dots can be continuously tuned from 2 to $10 \mathrm{~nm}$, which, after polymer encapsulation, generally increases to $5-20 \mathrm{~nm}$ in diameter. Particles smaller than $5 \mathrm{~nm}$ are quickly cleared by renal filtration. Semiconductor nanocrystals have unique and fascinating optical properties; become an indispensable tool in biomedical research, especially for multiplexed, quantitative and long-term fluorescence imaging and detection. QD core can serve as the structural scaffold, and the imaging contrast agent and small molecule hydrophobic drugs can be embedded between the inorganic core and the amphiphilic polymer coating layer. Hydrophilic therapeutic agents including small interfering RNA (siRNA) and antisense oligodeoxynucleotide (ODN)) and targeting biomolecules such as antibodies, peptides and aptamers can be immobilized onto the hydrophilic side of the amphiphilic polymer via either covalent or non-covalent bonds. This fully integrated nanostructure may behave like magic bullets that will not only identify, but bind to diseased cells and treat it. It will also emit detectable signals for real-time monitoring of its trajectory. 


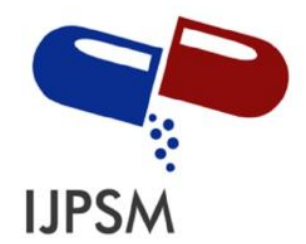

\section{Dr. M.Kannadasan et al, Int. Journal of Pharmaceutical Sciences and Medicine (IJPSM), Vol.5 Issue. 12, December- 2020, pg. 46-58}

ISSN: 2519-9889

Impact Factor: $\mathbf{3 . 4 2 6}$

\section{Superparamagnetic Nanoparticles}

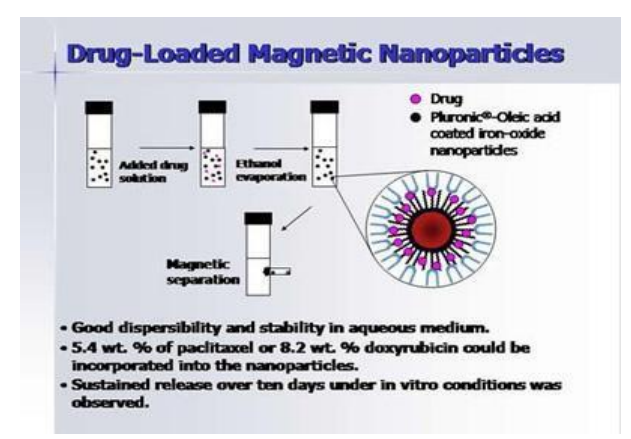

Figure 7: Superparamagnetic nanoparticles

Super paramagnetic molecules are those that are attracted to a magnetic field but do not retain residual magnetism after the field is removed. Nanoparticles of iron oxide with diameters in the 5-100 $\mathrm{nm}$ range have been used for selective magnetic bioseparations. Typical techniques involve coating the particles with antibodies to cell-specific antigens, for separation from the surrounding matrix. The main advantages of superparamagnetic nanoparticles are that they can be visualized in magnetic resonance imaging (MRI) due to their paramagnetic properties; they can be guided to a location by the use of magnetic field and heated by magnetic field to trigger the drug release. Super paramagnetic nanoparticles belong to the class of inorganic based particles having an iron oxide core coated by either inorganic materials (silica, gold) and organic (phospholipids, fatty acids, polysaccharides, peptides or other surfactants and polymers). In contrast to other nanoparticles, superparamagnetic nanoparticles based on their inducible magnetization, their magnetic properties allow them to be directed to a defined location or heated in the presence of an externally applied AC magnetic field. These characteristics make them attractive for many applications, ranging from various separation techniques and contrast enhancing agents for MRI to drug delivery systems, magnetic hyperthermia (local heat source in the case of tumor therapy), and magnetically assisted transfection of cells. Already marketable products, so-called beads, are micron sized polymer particles loaded with SPIONs.

\section{HISTORY ${ }^{1.2 .3}$}

By year 2050, human population will reach 9,100 million which is about $34 \%$ increase in population from present situation. By increasing in this population, it proportionally increases in global demand for foods, feed and energy. Despite on the expected demand on food (and water), several technology should beapplied to make a rational use of resources possible. Considering to this situation, nanotechnology could suppose a great tool in solving that demand. Exploration of nanotechnology has brought significantly innovations to the pharmacology fields for over past 30 years. Engineered nanomaterials (ENMs), one of nanotechnology application already became part of human daily life as food packaging agents, drug delivery systems, therapeutics, biosensors, and many more. By European Parliament and Council definition, 'nanodimension $\leq 100 \mathrm{~nm}$, or comprises of separate functional parts either internal or on the surface, which have one or more dimensions $\leq 100 \mathrm{~nm}$, which include structures, in example, agglomerates or aggregates, which may be larger than $100 \mathrm{~nm}$, but will retain the typical properties of nanoscale . FAO/WHO report [5] that the ENMs have several applications in the agrofood sector include nanostructured food ingredients, nanodelivery systems, organic and inorganic nanosized additives, nanocoatings on food contact surfaces, surface functionnalized NMs, nanofiltration, nanosized agrochemicals, nanosensors, water 


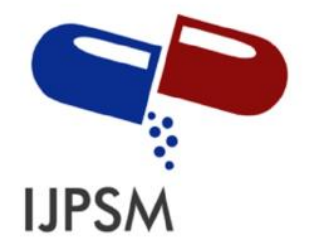

\section{Dr. M.Kannadasan et al, Int. Journal of Pharmaceutical Sciences and Medicine (IJPSM), Vol.5 Issue. 12, December- 2020, pg. 46-58}

ISSN: 2519-9889

Impact Factor: 3.426

decontamination, etc. However, in this chapter, authors will highlight on nanodelivery system from history up to its application.

Nanoscale size nanostructures are able to penetrate tissues and are easily taken up by cells, allowing for efficient delivery of drugs to target sites of action. Uptake of nanostructures has been reported to be 15-250 times greater than that of micro particles in the 1-10 $\mu \mathrm{m}$ range. Nanotechnology improves performance and acceptability of dosage forms by increasing their effectiveness, safety, patient adherence, as well as ultimately reducing health care costs. It may also enhance the performance of drugs that are unable to pass clinical trial phases. Nanotechnology definitely promises to serve as drug delivery carrier of choice for the more challenging conventional drugs used for the treatment and management of chronic diseases such as cancer, asthma, hypertension, HIV and diabetes [7]

\section{NEED $^{7}$}

At present $95 \%$ of all new potential therapeutics have poor pharmacokinetic and biopharmaceutical properties. Therefore, there is a need to develop suitable drug delivery systems that distribute the therapeutically active drug molecule only to the site of action, without affecting healthy organs and tissues, also lowering doses required for efficacy as well as increasing the therapeutics indices and safety profiles of new therapeutics.

Different reasons are

1) Pharmaceutical

- Drug instability in conventional dosage form

- Solubility

- Biopharmaceutical Low absorption

- High membrane bounding

- Biological instability

3) Pharmacokinetic/ Pharmacodynamics

- Short half life

- Large volume of distribution

- Low specificity

4) Clinical

- Low therapeutic index

\section{Objective $^{7}$}

The major goals in designing nanoparticles as a delivery system are to control particle size, surface properties and release of pharmacologically active agents in order to achieve the site-specific action of the drug at the therapeutically optimal rate and dose regimen

To achieve a desired pharmacological response at a selected site without undesirable interactions at other site, thereby the drugs have a specific action minimum side effects $\&$ better therapeutic index.

Ex: in cancer chemotherapy \& Enzyme replacement therapy.

\section{Ideal Characteristics ${ }^{7}$}

Targeted drug delivery system should be

Biochemically inert (non-toxic), non-immunogenic

Both physically \& chemically stable in vivo \& in vitro. 


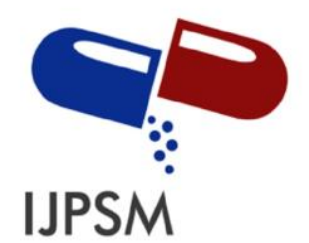

\section{Dr. M.Kannadasan et al, Int. Journal of Pharmaceutical Sciences and Medicine (IJPSM), Vol.5 Issue. 12, December- 2020, pg. 46-58}

ISSN: 2519-9889

Impact Factor: $\mathbf{3 . 4 2 6}$

Restrict drug distribution to target cells (or) tissues (or) organs \& should have uniform capillary distribution.

Controllable \& predicate rate of drug release.

Drug release does not effect drug action.

Therapeutic amount of drug release.

Minimal drug leakage during transit.

Carriers used must be biodegradable (or) readily eliminated from the body without any problem \& no carrier induced modulation of diseased state. The preparation of the delivery system should be easy (or) reasonably simple reproductive \& cost effective.

\section{Methods of Preparation of Nanoparticles ${ }^{7}$}

Nanoparticles can be prepared from a variety of materials such as proteins, polysaccharides and synthetic polymers. The selection of matrix materials is dependent on various factors which include:
a. Size of nanoparticle required
b. Inherent properties of the drug, e.g., aqueous solubility and stability
c. Surface characteristics such as charge and permeability
d. Degree of biodegradation, biocompatibility and toxicity
e. Drug release profile desired
f. Antigenicity of the final product.

\section{Methods of preparation}

The various methods are as follows:

1. Emulsion Polymerization

2. Desolvation method

3. High Pressure Homogenization

4. Controlled Gellification Method

5. Controlled Nanoprecipitation without Surfactants

6. Solvent Evaporation Method

7. Solvent Emulsification or Solvent Diffusion method

8. Supercritical Fluid Extraction

9. Melt Emulsification and Homogenization following Spray drying of nanodispersions.

\section{Mechanism of Nano delivery inside human body [11]}

One of example that has been proposed by was about the mechanism of alginate-enclosed chitosan-calcium phosphate-iron-saturated bovine lactoferrin nanocarrier (AEC-CP-Fe-bLf NCs) internalization and its action inside human body. From the figure, it shows that the alginate coating of orally directed AEC-CP-Fe-bLf NCs is degraded in the alkaline environment offered in the small intestine

(A). Then, the alginate coating free C-CP-Fe-bLf NCs enter the blood circulation via endocytosis and/or transcytosis (B). After that, C-CP-Fe-bLf NCs are released in the tumor site by making use of the enhanced permeability retention effect(C). Finally, the uptake of C-CP-Fe-bLf NCs in to the cancer cells is based on oligosaccharide and/or lactoferrin receptor-mediated endocytosis(D). 


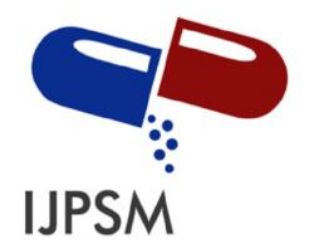

\section{Dr. M.Kannadasan et al, Int. Journal of Pharmaceutical Sciences and Medicine (IJPSM), Vol.5 Issue. 12, December- 2020, pg. 46-58}

ISSN: 2519-9889

Impact Factor: 3.426

\section{Evaluation of Nanoparticles ${ }^{8,9}$ Zeta potential}

Zeta potential of a nanoparticle is commonly used to characterize the surface charge property of nanoparticles. The reflects the electrical potential of particles and is influenced by the composition of the particle and the medium in which it is dispersed. Nanoparticles with a zeta potential above $( \pm) 30 \mathrm{mV}$ have been shown to be stable in suspension, as the surface charge prevents aggregation of the particles.

\section{Particle Shape ${ }^{[17]}$}

SEM characterizes the nanosuspension before going for evaluation; the nanosuspension is lyophilized to form solid particles. The solid particles are coated with platinum alloy using a sputter coater.

\section{Particle size ${ }^{[18]}$}

Particle size and size distribution are the most important characteristics of nanoparticle systems. They determine the in vivo distribution, biological fate, and toxicity and targeting ability of nanoparticle system. In addition, they can also influence the drug loading, drug release and stability of nanoparticles. Currently, the faster and most routine method of determining particle size is by photon-correlation spectroscopy or dynamic light scattering. The results obtained by photon-correlation spectroscopy are usually verified by scanning or transmission electron microscopy (SEM or TEM).

\section{Drug Entrapments Efficiency ${ }^{9}$}

The nanoparticles were separated from the aqueous medium by ultracentrifugation at $10,000 \mathrm{rpm}$ for $30 \mathrm{~min}$ at 50C. Then the resulting supernatant solution was decanted and dispersed into phosphate buffer saline $\mathrm{pH}$ 7.4. Thus the procedure was repeated twice to remove the unentrapped drug molecules completely. The amount of drug entrapped in the nanoparticles was determined as the difference between the total amount of drug used to prepare the nanoparticles and the amount of drug present in the aqueous medium.

Drug Entrapment efficiency $(\%)=$ Amount of released from the lysed nanoparticle X 100 Amount of drug initially taken to prepare the Nanoparticles

\section{Advantages of Nanoparticles ${ }^{9,10}$}

The advantages of using nanoparticles as a drug delivery system include the following:

1) Particle size and surface characteristics of nanoparticles can be easily manipulated to achieve both passive and active drug targeting after parenteral administration.

2) They control and sustain release of the drug during the transportation and at the site of localization, altering organ distribution of the drug and subsequent clearance of the drug so as to achieve increase in drug therapeutic efficacy and reduction in side effects.

3) Site-specific targeting can be achieved by attaching targeting ligands to surface of particles or use of magnetic guidance.

4) Controlled release and particle degradation characteristics can be readily modulated by the choice of matrix constituents. Drug loading is relatively high and drugs can be incorporated into the systems without any chemical 


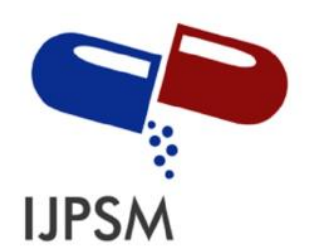

\section{Dr. M.Kannadasan et al, Int. Journal of Pharmaceutical Sciences and Medicine (IJPSM),} Vol.5 Issue. 12, December- 2020, pg. 46-58

ISSN: 2519-9889

Impact Factor: 3.426

reaction; this is an important factor for preserving the drug activity.

5) The system can be used for various routes of Administration including oral, nasal, parenteral, intra-ocular etc.

\section{Limitations of Nanoparticles}

Small size and large surface area can lead to particleparticle aggregation, making physical handling of nanoparticles difficult in liquid and dry forms. In addition, small particles size and large surface area readily result in limited drug loading and burst release. These practical problems have to be overcome before nanoparticles can be used clinically or made commercially available.

\section{Applications of Nanoparticles: ${ }^{10}$}

\section{Tumor targeting using Nanoparticulate delivery system}

The rational of using nanoparticles for tumor targeting is based on (1) Nanoparticles will be able to deliver a concentrate dose of drug in the vicinity of the tumor targets via the enhanced permeability and retention effect or active nanoparticles. (2) Nanoparticles will reduce the drug exposure of health tissues by limiting drug distribution to target organ.An experiment demonstrated in mice treated with doxorubicin incorporated into poly (isohexylcynoacrylate) nanospheres that higher concentration of doxorubicin manifested in the liver, spleen and lungs than in mice treated with free doxorubicin.

\section{Nanoparticles for Gene delivery}

Polynucleotide vaccines work by delivering genes encoding relevant antigens to host cells where they are expressed, producing the antigenic protein within the vicinity of professional antigen presenting cells to initiate immune response. Such vaccines produce both humoral and cell-mediated immunity because intracellular production of protein, as opposed to extracellular deposition, stimulates both arms of the immune system.

\section{Nanotechnology in Medicine Application:}

Anti-Microbial Techniques

One of the earliest nanomedicine applications was the use of nanocrystalline silver, which is as an antimicrobial agent for the treatment of wounds, A nanoparticle cream has beenshown to fight staph infections. The nanoparticles contain nitric oxide gas, which is known to kill bacteria. Studies on mice have shown that using the nanoparticle cream to release nitric oxide gas at the site of staph abscesses significantly reduced the infection. Burn dressing that is coated with nanocapsules containing antibotics. If an infection starts the harmful bacteria in the wound causes the nanocapsules to break open, releasing the antibotics. This allows much quicker treatment of an infection and reduces 


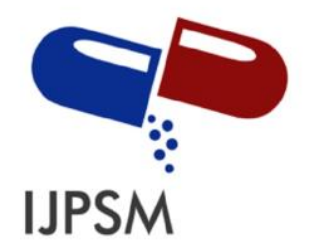

\section{Dr. M.Kannadasan et al, Int. Journal of Pharmaceutical Sciences and Medicine (IJPSM), Vol.5 Issue. 12, December- 2020, pg. 46-58}

ISSN: 2519-9889

Impact Factor: 3.426

the number of times a dressing has to be changed. A welcome idea in the early study stages is the elimination of bacterial infections in a patient within minutes, instead of delivering treatment with antibiotics over a period of weeks.

\section{Nanotechnology in Medicine Application: Cell Repair}

Nanorobots could actually be programmed to repair specific diseased cells, functioning in a similar way to antibodies in our natural healing processes. Read about design analysis for one such cell repair nanorobot in this article:

The Ideal Gene Delivery Vector: Chromallocytes, CellRepair Nanorobots for Chromosome Repair Therapy. Nanoparticles for drug delivery into the brain

The blood-brain barrier (BBB) is the most important factor limiting the development of new drugs for the central nervous system. Relatively impermeable endothelial cells characterize the BBB with tight junctions, enzymatic activity and active efflux transport systems. It effectively prevents the passage of water-soluble molecules from the blood circulation into the CNS, and can also reduce the brain concentration of lipid-soluble molecules by the function of enzymes or efflux pumps. Consequently, the BBB only permits selective transport of molecules that are essential for brain function. Strategies for nanoparticle targeting to the brain rely on the presence of and nanoparticle interaction with specific receptor-mediated transport systems in the BBB. For example polysorbate 80/LDL, transferrin receptor binding antibody (such as OX26), lactoferrin, cellpenetrating peptides and melanotransferrin have been shown capable of delivery of a self non transportable drug into the brain via the chimeric construct that can undergo receptor-mediated transcytosis. It has been reported poly(butylcyanoacrylate) nanoparticles was able to deliver hexapeptidedalargin, doxorubicin and other agents into the brain which is significant because of the great difficulty for drugs to cross the BBB. Despite some reported success with polysorbate 80 coated NPs, this system does have many shortcomings including desorption of polysorbate coating, rapid NP degradation and toxicity caused by presence of high concentration of polysorbate 80. OX26MAbs (anti-transferrin receptor MAbs), the most studied BBB targeting antibody, have been used to enhance the BBB penetration of lipsosomes. However, recently, Jiet al. demonstrated that brain uptake of lactoferrin, an iron-binding glycoprotein belonging to the transferrin (Tf) family, is twice that of OX26 and transferrrinin vivo. 


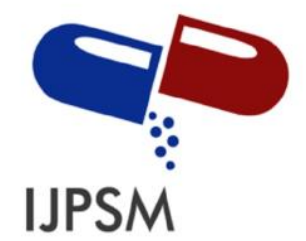

Dr. M.Kannadasan et al, Int. Journal of Pharmaceutical Sciences and Medicine (IJPSM), Vol.5 Issue. 12, December- 2020, pg. 46-58

ISSN: 2519-9889

Impact Factor: 3.426

\section{NEW APPROCHES ${ }^{6}$}

\section{Nanodrug delivery systems for anti-cancer agents}

Many researchers have used different approaches and techniques for formulating nanoparticles for anti-cancer agents. Some of these studies along with their prominent findings are mentioned here.

TABLE 1: Different types of anti-cancer agents and their mode of action against cancer cell

\begin{tabular}{|c|c|c|c|c|c|c|c|}
\hline \multicolumn{4}{|c|}{ Hydrophobic properties } & \multicolumn{4}{|c|}{ Hydrophilic properties } \\
\hline $\begin{array}{l}\text { Anti- } \\
\text { cancer } \\
\text { agents } \\
\end{array}$ & $\begin{array}{l}\text { Techniq } \\
\text { ues }\end{array}$ & $\begin{array}{l}\text { Mode of } \\
\text { actions }\end{array}$ & Findings & $\begin{array}{l}\text { Anti- } \\
\text { cancer } \\
\text { agents } \\
\end{array}$ & Techniques & $\begin{array}{l}\text { Mode of } \\
\text { actions }\end{array}$ & Findings \\
\hline $\begin{array}{l}\text { Paclitax } \\
\text { el }\end{array}$ & $\begin{array}{l}\text { Albumin } \\
\text {-bound } \\
\text { paclitaxe } \\
1 \text { (ABI- } \\
007 \text {, } \\
\text { Abraxan } \\
\text { e®; } \\
\text { Abraxis } \\
\text { BioScien } \\
\text { ce and } \\
\text { AstraZen } \\
\text { eca). }\end{array}$ & $\begin{array}{l}\text { natural } \\
\text { carrier of } \\
\text { endogenous } \\
\text { hydrophobic } \\
\text { molecules } \\
\text { such as } \\
\text { vitamins, } \\
\text { hormones } \\
\text { and other } \\
\text { water- } \\
\text { insoluble } \\
\text { plasma } \\
\text { substances. }\end{array}$ & $\begin{array}{l}\text { help } \\
\text { endothe } \\
\text { lial } \\
\text { transcyt } \\
\text { osis of } \\
\text { protein- } \\
\text { bound } \\
\text { and } \\
\text { unboun } \\
\text { d } \\
\text { plasma } \\
\text { constitu } \\
\text { ents }\end{array}$ & $\begin{array}{l}\text { Pluron } \\
\text { ics }\end{array}$ & $\begin{array}{l}\text { Incorporated with } \\
\text { doxorubicin and } \\
\text { other anticancer } \\
\text { agents }\end{array}$ & $\begin{array}{l}\text { interact } \\
\text { with multi- } \\
\text { drug } \\
\text { resitance } \\
\text { (MDR) } \\
\text { cancer cells }\end{array}$ & $\begin{array}{l}\text { drastic } \\
\text { sensitizat } \\
\text { ion of } \\
\text { cancer } \\
\text { cell }\end{array}$ \\
\hline
\end{tabular}

Some Indian Technologies

1. First produced smart hydrogel nanoparticles for drug delivery applications(US Patent 5847111)

2. Tumor Targeted Delivery of Taxol using nanoparticles (US Patent 6,322,817)

3. Inorganic Nanoparticles as non-viral vectors for targeted delivery of genes (US Patent 6555376 ); Technology transferred to a California based Pharm Com

4. Once in 48 hours dose Ophthalmic delivery (US Patent 6579519) (Another improved formulation patent on ophthalmic gels is being submitted in India)

5. Oral Insulin Delivery (Patent Pending)

\section{Conclusion}

Nanoparticles represent a promising drug delivery system of controlled and targeted release. The emergence of nanotechnology is likely to have a significant impact on drug delivery sector, affecting just about every route of administration from oral to injectable. In addition, the payoff for doctors and patients should be lower drug toxicity, reduced cost of treatments, improved bioavailability, and an extension of the economic life of proprietary drugs. The foregoing show that nano particulate systems have great potentials, being able to convert poorly soluble, poorly absorbed and labile biologically active substance into promising deliverable drugs. The core of this system can enclose a variety of drugs, enzymes, genes and is characterized by a long due to the hydrophilic shell which prevents recognition by the reticular-endothelial system To optimize this drug delivery system, greater understanding of the 


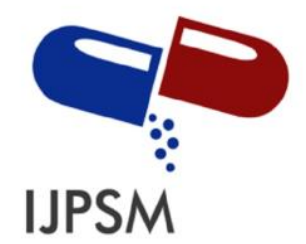

\section{Dr. M.Kannadasan et al, Int. Journal of Pharmaceutical Sciences and Medicine (IJPSM), Vol.5 Issue. 12, December- 2020, pg. 46-58}

ISSN: 2519-9889

Impact Factor: 3.426

different mechanisms of biological interactions, and particle engineering, is still required. Further advances are needed in order to turn the concept of nanoparticles technology into a realistic practical application as the next generation of drug delivery system. This would allow earlier and more personalized diagnosis and therapy, improving the effectiveness of drug treatments and reducing side effects. In addition, nanoparticles are a promising platform technology for the synthesis of molecular-specific contrast agents.

\section{REFERENCES}

1) Francisco J G., Ma L M., Paloma G., Ruth R. Nanotechnology and Food Industry. In: Dr. Benjamin Valdez. (Ed.) Scientific, Health and Social Aspects of the Food Industry. ISBN: 978-953-307-916-5: InTech; 2012. p 1-35

2) Michele F, Oliveira \& Pedro P G, Guimarães \& Alinne D M, Gomes \& Diego S, Rubén D S. Strategies to target tumors

3) Delvecchio Rick. Berkeley considering need for nano safety.articles.sfgate.com; 2006.

4) Langer R. Biomaterials in drug delivery and tissue engineering; one labortory's experience. Acc ChemRes.2000; 33:94-101.

5) Bhadia D, Bhadra S, Jain P and Jain NK. Pegnology; a review of PEGylated systems; Pharmazin. 2002; 57:5-20.

6) Kommaleddy S, Tiwari SB and Amiji MM. Long circulating polymeric nanovectors for tumour selective gene delivery technol. cancer Res Treat. 2005;4:615-25

7) Theresa Phillipos. Nanoparticles safe! About .co. Guide; 2009.

8) Cincinnati, OH, Approaches to safe Nano-technology; an iformation exchange with NIOSH; 2006, www.(dc.gov/niosh/topics/nano/exchange.hmt.)

9) Cho K, Wang X, Nie $S$, et al. Therapeutic nanoparticles for drug delivery in cancer. Clin

10) Cancer Res 2008; 14:1310-1316.

\section{Authors Biography:}

Dr. M.Kannadasan, Professor \& Principal, School of Pharmaceutical Sciences, Pratap University, Jaipur, Rajasthan, has been awarded the degree of Master of Pharmacy from Sagar University, is Pioneer in Pharmaceutical Education and Research across Globe, Now, rechristened: Dr. H. S. Gour Central University, Sagar, India. He has Total Pharmaceutical Professional Experience of 18.5 Years (Teaching- ResearchAdministration). He authored review and research articles in peer-reviewed journals. Dasan has served as Head (i/c) in Dept. of Pharmacy, Agra University, and Agra. He did Technical Service for a short stint at Dept. of Pharmacology \& Toxicology, NIPER-Mohali. Dasan is a professional life member of ten official professional bodies in India, including his current fellowship with Institution of Chemist India, and has one more credit at international level: ATINER-Greece. Dasan is an Editorial and Reviewer Board Member in Seven National / International Journals. He also earned 17 Certificates from MOOC at SDG Academy \& Edx. He has been served as Director (Pharmacy) at SSITM, Aligarh, UP

Mr. Prem Kumar Bichala, Associate Professor, School of Pharmaceutical Sciences, Pratap University, Jaipur, Rajasthan, has been awarded the degree of Master of Pharmacy in Pharmaceutical Analysis by Acharya Nagarjuna University, Guntur, A.P. Presently pursuing Ph.D. from JNT University Kakinada, Kakinada, A.P. He has 10 years of teaching and research experience. He has published various research papers in peer reviewed National \& International journals.

Mr. Abhishek Agrawal, Assistant Professor, School of Pharmaceutical Sciences, Pratap University, Jaipur, Rajasthan, has been awarded the degree of Master of Pharmacy in Pharmaceutical Chemistry from Rajiv Academy for Pharmacy, Mathura. He has 7 years of teaching and research experience.

Ms. Swapna Singh, Assistant Professor, School of Pharmaceutical Sciences, Pratap University, Jaipur, Rajasthan, has been awarded the degree of Master of Pharmacy in Pharmaceutical Chemistry from Technocrat institute of technology-Pharmacy, Bhopal. She has 2 years of teaching and research experience. 